ISSN 0001-6012/2017/59/3/103-109 Acta Médica Costarricense, () 2017 Colegio de Médicos y Cirujanos de Costa Rica

\title{
Opinión
}

\section{Guías de manejo de artritis reumatoide Consenso 2016}

\section{Asociación Costarricense de Reumatología}

(Management Guidelines of Rheumatoid Arthritis Consensus 2016)

Raúl Alpízar-Campos', José Francisco Díaz-Coto², José Manuel Vega-Ortiz², Pablo Monge-Zeledón², Dina ArrietaVega $^{2}$ y Ricardo Sáenz-Castro ${ }^{4}$

\section{Resumen}

La artritis reumatoide sigue representando un importante factor de deterioro de la salud de las personas que sufren esta condición, a pesar de los avances recientes en su tratamiento. En forma adicional, se reconoce su significativo impacto a nivel social y económico. La Asociación Costarricense de Reumatología publicó en 2010, las primeras guías de manejo de esta enfermedad. En el presente trabajo, este primer documento fue actualizado, mediante la revisión exaustiva de la información científica disponible y relevante a la fecha, con el objetivo de uniformar y optimizar la atención de la artritis reumatoide en Costa Rica. Incluye las recomendaciones de evaluación de los pacientes, su seguimiento y tratamiento. También se revisan las opciones de medicamentos disponibles en el país, es decir, drogas modificadoras de los síntomas y fármacos modificadores de la enfermedad convencionales, además de agentes biológicos y moléculas pequeñas.

Descriptores: artritis reumatoide, guías de manejo, clinimetría, terapia biológica.

\section{Abstract}

Rheumatoid arthritis continues to represent an important factor of deterioration in the health of people suffering from this condition despite recent medical advances in their treatment. Additionally, its significant social and economic impact it is recognized. The Costa Rican Association of Rheumatology published in 2010 the first guidelines for the management of this disease. In the present work, this first document is updated, through an exhaustive review of the available and relevant scientific information to date. This review aims to standardize and optimize the care of rheumatoid arthritis in Costa Rica. It includes recommendations for evaluation of patients, follow-up and treatment. There is also a review of drug options available in the country, i.e., symptoms-modifying drugs, conventional disease modifying drugs, as well as biological agents and small molecules.

Keywords: rheumatoid arthritis, management guides, clinimetrics, biological therapy.

Fecha recibido: 02 de marzo de 2017

Fecha aprobado: 20 de abril de 2017

La artritis reumatoide (AR) es una enfermedad inflamatoria crónica, que se estima afecta aproximadamente del 0,5\% al $1 \%$ de la población general. Dado su compromiso articular progresivo y afectación sistémica, esta condición conlleva a un significativo deterioro de la salud y consumo de recursos con su consecuente impacto socioeconómico. 
Con la introducción de nuevos enfoques de tratamiento y opciones terapéuticas, diferentes países del mundo y sociedades médicas han creado en los últimos años, guías de manejo o recomendaciones de tratamiento, las cuales pretenden uniformar y mejorar la atención de los pacientes con AR. En Costa Rica, la Asociación Costarricense de Reumatología publicó las primeras guías nacionales en 2010, procurando adecuar el manejo de esta enfermedad al contexto de la realidad nacional.

No obstante, en los últimos años ha habido importantes avances científicos en Reumatología y se han establecido nuevos paradigmas en el campo del tratamiento de la AR, razón por la cual la Asociación ha tomado la iniciativa de actualizar la propuesta inicial de 2010, incorporando la información científica disponible a la fecha.

\section{Metodología}

Dado que se contaba con guías previas, las cuales en su momento habían tenido una aprobación de más del $80 \%$ de los asociados, se consideró solamente proceder con una revisión de los aspectos relevantes que habían presentado cambios en los últimos 6 años, a la luz del conocimiento científico disponible.

Para actualizar las guías de manejo de AR, se escogió una modificación del método Delphi, y en asamblea general de la Asociación se establecieron 5 grupos de trabajo con diferentes temas. Cada grupo de trabajo debería investigar toda la bibliografía existente y relevante en su área para luego discutir la información recopilada y producir un documento con las recomendaciones de cambio a las guías existentes, con modificaciones concretas y claras. Los temas de los grupos de trabajo fueron:

1. Definiciones y generalidades en artritis reumatoide

2. Clinimetría y evaluación clínica de la enfermedad

3. Glucocorticoides y drogas antiinflamatorias no esteroideas (AINEs)

4. Fármacos modificadores de la enfermedad (FARMEs) convencionales

5. Agentes biológicos y moléculas pequeñas

6. Consideraciones sobre embarazo y artritis reumatoide

En una segunda fase del proyecto, las recomendaciones de cambio propuestas por cada grupo de trabajo fueron presentadas, revisadas y sometidas a votación en una asamblea general, convocada exclusivamente para este objetivo. Se estableció que se debería contar con un grado de consenso mínimo del 80\% para incluir el ítem de cambio en la nueva versión de las guías de manejo para artritis reumatoide.

\section{Resultados}

De los ítems formulados, se aprobó el 95\%.

\section{Generalidades:}

Se considera que el manejo actual de esta enfermedad tiene dos principios básicos: 1 . Concebir la enfermedad como una condición grave, pero eventualmente modificable, 2. El control estricto de la enfermedad requiere la participación de un médico reumatólogo, quien posee una visión y concepto global de la enfermedad, además de dominar los principios farmacológicos de las drogas modificadoras y los parámetros de evaluación clínica del paciente.

Se encuentra establecido que la AR es una enfermedad autoinmune, cuyo mecanismo fisiopatológico básico es la inflamación sinovial constante, que resulta en la erosión articular.

Se considera AR temprana, a aquella enfermedad de menos de 12 semanas de evolución, periodo durante el cual se comporta diferente desde el punto de vista inmunológico y patológico, en comparación con una enfermedad más tardía. El inicio de tratamiento en la etapa temprana ha mostrado mayores tasas de remisión a los 6 meses y mejor pronóstico clínico y radiológico a los 3 años, lapso que se conoce como ventana de oportunidad terapéutica. Adicionalmente, se ha catalogado como AR muy temprana a aquella enfermedad de menos de 8 semanas.

Los criterios de clasificación del Colegio Americano de Reumatología (ACR)/ Liga Europea contra el Reumatismo (EULAR), vigentes para artritis reumatoide temprana, se exponen en el Cuadro 1.

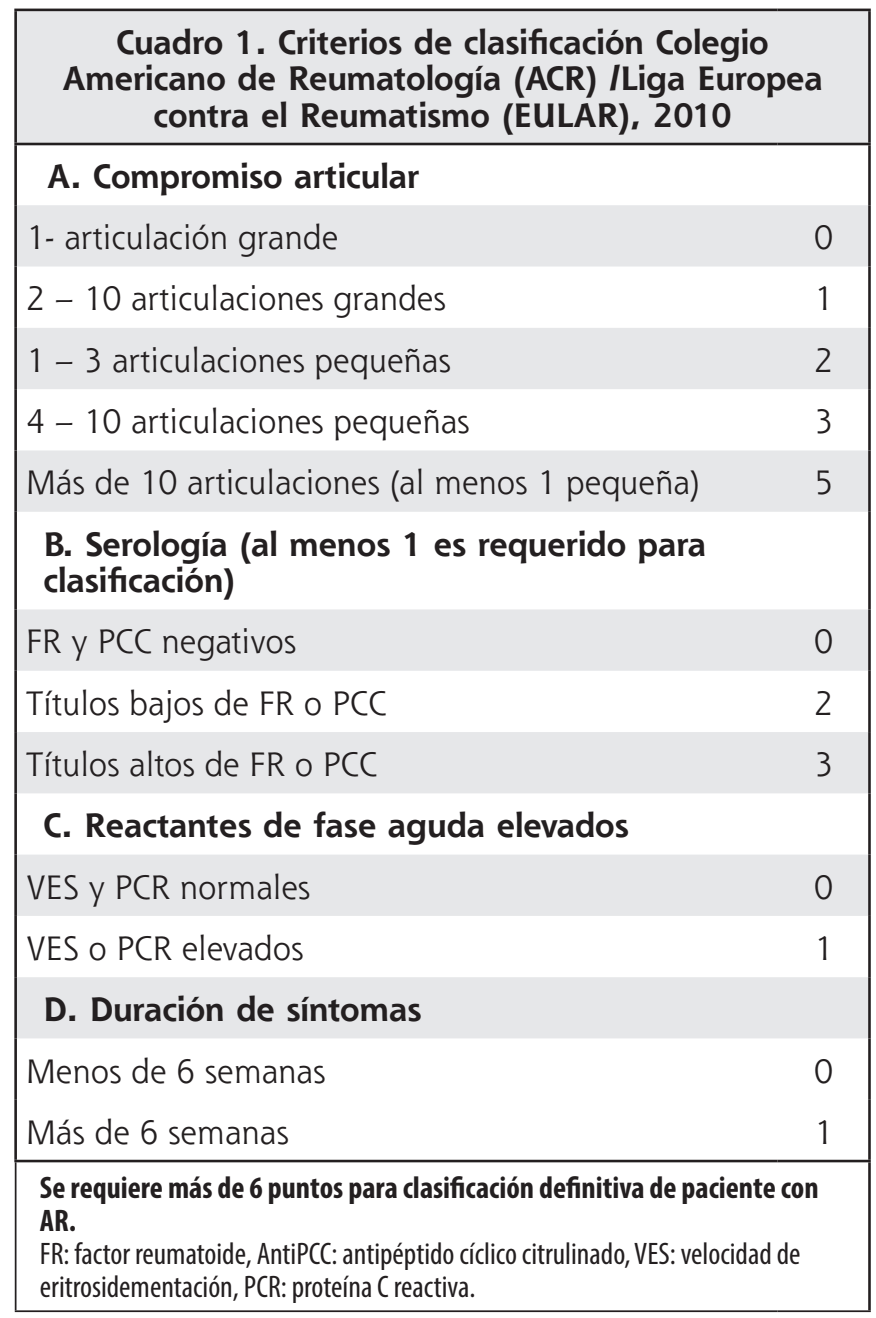


Guías de manejo de artritis reumatoide / Alpízar-Campos et al

El principal objetivo del tratamiento en el paciente con AR o meta terapéutica es la remisión de la enfermedad, o al menos alcanzar un bajo nivel de actividad clínica. Esta estrategia busca mejorar los síntomas y signos, disminuir la progresión del daño articular, prevenir la limitación funcional y mejorar la calidad de vida. La valoración se realizará mediante métodos clinimétricos estandarizados. El control estricto de la enfermedad permite alcanzar la meta terapéutica.

Se define como falla terapéutica completa, la falta de respuesta al tratamiento utilizado, es decir, que no haya alcanzado la meta terapéutica con la terapia combinada a dosis máximas toleradas, por un periodo no menor de tres meses. La respuesta terapéutica incompleta es aquella en la que se ha logrado una reducción parcial, pero sin alcanzar la meta terapéutica.

La definición de artritis refractaria se aplica a aquellos pacientes con falla terapéutica a por lo menos dos combinaciones de FARMEs aprobadas, o a aquellos pacientes que no han podido completar el esquema señalado, debido a efectos adversos a los medicamentos.

Los factores de mal pronóstico en esta condición son: la presencia de daño radiológico al momento del diagnóstico, sexo femenino, elevación de VES y PCR, presencia de factor reumatoide y péptido citrulinado cíclico en títulos elevados. Todos estos factores al momento del diagnóstico, predicen la progresión del daño articular en el tiempo.

Efectuado el diagnóstico, se recomienda que los pacientes sean evaluados cada mes durante los primeros meses; al momento de alcanzar la remisión sostenida, las evaluaciones médicas pueden programarse para entre tres y seis meses.

\section{Clinimetría y evaluación clínica de la enfermedad}

La remisión es un término utilizado para establecer un estado libre de enfermedad. En la AR este concepto se encuentra relacionado con tres aspectos fundamentales: ausencia de actividad inflamatoria articular, ausencia de progresión radiológica de daño articular y conservación de la capacidad funcional.

Desde el punto vista de la práctica clínica diaria, para determinar la ausencia de actividad inflamatoria por artritis, se recomienda utilizar un método de evaluación combinada. Los métodos de evaluación combinada incluyen las siguientes variables: valoración global de la enfermedad por parte del paciente, valoración de la enfermedad por parte del médico, número de articulaciones dolorosas, número de articulaciones inflamadas y los valores de los reactantes de fase aguda (VES o PCR). Es importante tomar en cuenta que el proceso de recolección de los datos para estas escalas debe realizarse siempre en forma sistemática y homogénea, lo que permitirá evaluaciones más objetivas y establecer con claridad la evolución de un paciente en el tiempo.

Los métodos de evaluación combinada más utilizados son: DAS 28, CDAI y SDAI. Ofrecen un valor numérico que

permiten estratificar la actividad inflamatoria en rangos tales como: actividad alta, actividad moderada, actividad baja y remisión. (Cuadro 2)

La determinación de remisión se hará de acuerdo con alguna de las siguientes definiciones: $\mathrm{DAS}_{28} \leq 2,6-$ SDAI $\leq 3,3-$ CDAI $\leq 2,8$. El DAS 28 debe de ser de cuatro variables. Además, se considera que la valoración global por parte del paciente permite, en cierta medida, valorar las articulaciones que no están incluidas en el recuento de las 28 establecidas. En pacientes con $\mathrm{DAS}_{28} \leq 2,6$, con más de dos articulaciones inflamadas, debe considerarse la valoración de éstas por ultrasonografía, con el fin de confirmar la presencia de sinovitis activa, a través de al menos un power Doppler mayor a grado 2 .

El impacto de la enfermedad en el paciente debe ser evaluado al menos una vez al año, utilizando el Health Assessment Questionaire (HAO). Este instrumento permite determinar la condición del paciente y su grado de limitación funcional, y también predice el daño estructural, la discapacidad y el riesgo de muerte.

El daño estructural se estima a través de radiología convencional, al menos 1 vez al año, realizando un estudio basal y utilizando proyecciones PA de ambas manos, y dorso plantar de ambos pies, con foco en antepié. El método más recomendado es el Sharp-Van der Heijde, el cual evalúa la disminución del espacio articular y la presencia de erosiones.

Las recomendaciones del uso del ultrasonido en pacientes con AR, son las siguientes:

\begin{tabular}{|ccc|}
\hline Cuadro 2. Grado de actividad, según los diferentes \\
índices combinados
\end{tabular}


a. Pacientes en remisión clínica $\left(\mathrm{DAS}_{28} \leq 2,6\right)$, sin articulaciones inflamadas o dolorosas, en que se desee hacer una reducción de la dosis de mantenimiento; se valorarán las articulaciones de las muñecas y las II y III metacarpofalángicas (MCF).

b. Pacientes en remisión clínica ( $\left.\mathrm{DAS}_{28} \leq 2,6\right)$, con alguna articulación inflamada o dolorosa; se valorarán las articulaciones de las muñecas, las II y III MCF y las articulaciones con sinovitis residual por clínica.

c. Pacientes con $\mathrm{DAS}_{28} \geq 2,6$ a expensas de VES elevada; se valorarán las articulaciones de las muñecas y las II, III MCF, codos, rodillas y tobillos.

Los hallazgos en power Doppler grado II se consideran como sinovitis activa. Los hallazgos en escala de grises aislados se consideran como una secuela y no traducen actividad de la enfermedad. Los hallazgos en power Doppler grado 1, quedan a criterio del clínico, el cual deberá considerar el número de articulaciones comprometidas y los hallazgos en la escala de grises.

\section{Glucocorticoides (GC) y drogas antiinflamatorias no esteroideas (AINEs)}

Se considera que los AINEs son drogas útiles para controlar el dolor en la artritis reumatoide, y deben utilizarse en la dosis efectiva más baja, ya sea de forma continua o bajo demanda. Es necesario tomar en cuenta efectos secundarios de estos medicamentos en la práctica diaria; no obstante, pueden utilizarse en pacientes sintomáticos una vez que sus condiciones gastrointestinal, renal y cardiovascular hayan sido evaluadas cuidadosamente. Tanto para los DAINEs como para el paracetamol, se recomienda evitar su uso en presencia de hepatopatía crónica o enfermedad renal significativa.

En aquellos casos en los cuales se considere que exista riesgo cardiovascular, tanto los AINEs no selectivos como los COX-2 selectivos se deben usar con precaución a la dosis más baja efectiva y por el periodo más corto posible. Si la condición clínica lo amerita, se pueden usar AINEs junto con GC de vida intermedia, por el periodo más corto posible.

Hay poca evidencia que apoye el uso del paracetamol en AR; en caso de usarse, se recomienda no exceder una dosis máxima de 4 g/día.

Los GC pueden ser utilizados junto con los FARMEs en el abordaje terapéutico inicial, o bien, durante lapsos de crisis. La extensión de su uso estará en función del tiempo necesario para que los FARMEs alcancen su efecto terapéutico. Se recomienda una dosis de prednisona de 5 a 7,5 mg/día, de ser posible por un tiempo no mayor de seis meses.

En aquellos casos en los cuales se documente una crisis inflamatoria articular severa, se pueden utilizar los GC en forma oral o parenteral, a una dosis de prednisona de $0,5 \mathrm{mg} /$ $\mathrm{Kg} /$ día o su equivalente, con una posterior reducción semanal. Cuando existan manifestaciones extraarticulares severas, como la vasculitis reumatoide, los bolos intravenosos son una opción de tratamiento. A su vez, si se encuentra un número limitado de articulaciones inflamadas, se puede hacer uso de los GC intraarticulares de depósito, con un máximo de 3 o 4 procedimientos al año por articulación, con un intervalo no menor a 90 días entre procedimientos.

En caso de que se anticipe terapia esteroidal prolongada, se aconseja definir el riesgo de osteoporosis, tomando en cuenta la edad del paciente, estado postmenopáusico y el resultado de la densitometría ósea. Si se considera que existe riesgo de desarrollo de osteoporosis, es necesario completar el tratamiento con calcio, vitamina D y bifosfonatos.

\section{Fármacos modificadores de la enfermedad (FARMEs) convencionales:}

El tratamiento con FARMEs debe iniciarse tan pronto se establezca el diagnóstico de AR. El tratamiento inicial debe ser siempre con metotrexate a dosis máximas toleradas por el paciente, ya sea por vía parenteral u oral. La dosis recomendada es de 15 a 25 mg por semana. Dosis superiores de metotrexate a 17,5 mg por vía oral, se asocian con problemas de absorción, por lo que se puede dividir la dosis oral en dos días, para lograr una dosis máxima de $25 \mathrm{mg}$; en estos casos, se puede considerar el cambio a administración parenteral. Se recomienda asociar folatos de manera rutinaria con el uso de metotrexate.

En aquellos sujetos en los que no se pueda usar metotrexate, 0 ante la presencia de efectos adversos serios, hay evidencia que respalda el uso de sulfazalacina o leflunomida en monoterapia.

En los pacientes con AR con factores de mal pronóstico, debe iniciarse tratamiento combinado, al igual que en pacientes que no responden a monoterapia. Se considera que la leflunomida es tan efectiva como el metotrexate en monoterapia o en terapia combinada. En forma adicional, el uso de hidroxicloroquina se recomienda siempre en terapia combinada.

En el caso de la sulfasalazina, con el fin de mejorar su tolerancia, se sugiere iniciar con una dosis de $500 \mathrm{mg}$ al día, posteriormente aumentar la dosis en $500 \mathrm{mg}$ cada cinco a siete días, hasta lograr una dosis total diaria de dos a tres gramos.

Los esquemas de terapia combinada que han demostrado eficacia son: metotrexate más sulfasalazina más hidroxicloroquina, metotrexate más sulfasalazina, metotrexate más hidroxicloroquina y metotrexate más leflunomida (Cuadro 3).

\section{Agentes biológicos y otras moléculas pequeñas}

El uso de terapia biológica y otras moleculas pequeñas se debe considerar en pacientes con artritis reumatoide

\begin{tabular}{|cc|}
\hline \multicolumn{2}{|c|}{$\begin{array}{c}\text { Cuadro 3. Esquema terapéutico para artritis } \\
\text { reumatoide con FARMEs convencionales }\end{array}$} \\
\hline Monoterapia & Combinado \\
$\begin{array}{c}\text { Metotrexate (MTX) } \\
\text { Leflunomida }\end{array}$ & MTX + SSZ + Hidroxicloroquina \\
Sulfasalazina (SSZ) & MTX + Hidroxicloroquina \\
& MTX + SSZ \\
& MTX + Leflunomida \\
\hline
\end{tabular}


refractaria, es decir, aquellos que hayan utilizado por los menos dos esquemas recomendados de terapia combinada de FARMEs convencionales y en los cuales se haya documentado falla terapéutica, o que no pudieron completarlos por efectos adversos.

Las opciones de terapia biológica disponibles en el país son: agentes anti TNF (antifactor de necrosis tumoral), tocilizumab y rituximab (Cuadro 4). En el caso de moléculas pequeñas se cuenta con tofacitinib. La terapia biológica debe usarse idealmente de forma combinada con metotrexate $\mathrm{u}$ otro FARMEs convencional. En casos de intolerancia o alguna contraindicación a los FARMEs convencionales, se puede utilizar el tratamiento biológico en monoterapia. Solo el tocilizumab como agente biológico ha mostrado ser superior en monoterapia al metotrexate. No es recomendable la combinación de agentes biológicos. En forma adicional, los inhibidores de la JANUS quinasa, como el tofacitinib, también se puede usar ante falla a FARMEs.

Una vez definida la condición de artritis refractaria, se debe considerar el uso de terapia biológica o inhibidores de la JANUS quinasa. En este momento, se debe informar adecuadamente al paciente sobre la propuesta de cambio de tratamiento. La selección del agente biológico se realizará tomando en cuenta factores como: costo económico, comorbilidades del paciente, vía de administración, dosificación y eficacia. En aquellos casos en los cuales el paciente pueda cubrir el costo del tratamiento, se sugiere tomar en cuenta también su preferencia, dado que existen diferencias significativas en dosificación y administración, que pueden ser determinantes en términos de adherencia.

En los casos de los pacientes que fallen a la primera terapia biológica, puede modificarse su esquema de tratamiento y considerar un agente biológico con igual o distinto mecanismo de acción, pero se recomienda hacer el cambio a un fármaco con un mecanismo de acción diferente.

Conviene mencionar que la evidencia científica disponible confirma la eficacia de utilizar un segundo agente biológico en pacientes que han fallado a un antiTNF. No existen datos suficientes sobre la eficacia de un segundo biológico en pacientes que han fallado a biológicos diferentes de un antiTNF.

\begin{tabular}{|cc|}
\hline $\begin{array}{c}\text { Cuadro 4. Agentes biológicos disponibles en Costa } \\
\text { Rica para el tratamiento de la artritis reumatoide }\end{array}$ \\
\hline Tipo agente biológico & Nombre \\
& Infliximab \\
AntiTNF $\alpha$ & Etanercept \\
& Adalimumab \\
& Golimumab \\
AntiCD20 & Rituximab \\
AntilL-6 & Tocilizumab \\
\hline
\end{tabular}

\section{Consideraciones sobre embarazo y artritis reumatoide}

En las mujeres con AR y que deseen un embarazo, se recomienda que se encuentren en estado de remisión o baja actividad de la enfermedad, por un periodo de al menos tres meses previos a la concepción. Debe iniciarse suplemento con ácido fólico $5 \mathrm{mg}$ diarios, al menos tres meses antes de la concepción, y mantener la dosis a lo largo del embarazo.

La evaluación de la actividad de la enfermedad durante el embarazo debe realizarse únicamente con la escala de DAS 28 de 3 variables, utilizando PCR, hasta 3 meses postparto. No se recomienda utilizar la VES como parámetro clinimétrico, por la variación que este marcador presenta durante el embarazo. De la misma forma, no se aconseja usar la valoración global del paciente acerca de su enfermedad porque puede influenciarse por las molestias propias del embarazo.

En cuanto a las drogas que pueden administrarse durante el embarazo, los AINEs, no selectivos pueden usarse sin mayor riesgo durante los primeros dos trimestres. Se recomienda descontinuar el uso de AINEs a partir de la semana 32, por el riesgo de eventuales complicaciones. Los AINEs no selectivos son compatibles con la lactancia, pero se debe tomar en cuenta el riesgo potencial de ictericia y kernícterus.

No hay estudios que demuestren seguridad de los inhibidores COX-2 selectivos en embarazo, por lo que no se recomienda su uso en esta condición. En forma alternativa, se pueden utilizar los AINEs no selectivos en asociación con inhibidores de bomba de protones. En relación con inhibidores COX-2 y lactancia, los datos son insuficientes, por lo que no se recomiendan.

Los glucocorticoides no fluorinados se pueden utilizar durante el embarazo y se recomienda su uso cuando la terapia con FARMEs sea insuficiente para controlar la actividad. Los glucocorticoides deberían utilizarse a la menor dosis efectiva de prednisona (idealmente menos de $10 \mathrm{mg}$ diarios), por el menor tiempo posible. Los glucocorticoides son compatibles con la lactancia a las dosis usuales; se recomienda esperar 4 horas después de la administración de la última dosis del fármaco para iniciar la sesión de lactancia.

En relación con el uso de FARMEs, se considera que la hidroxicloroquina es segura durante el embarazo y la lactancia materna. La sulfasalazina puede utilizarse de forma segura durante el embarazo a una dosis máxima de 2 gramos al día. El metotrexate se debe suspender tres meses previos a la concepción y no es compatible con la lactancia. La leflunomida debe suspenderse al menos 6 meses antes de la concepción y deberá realizarse lavado con colestiramina (8 gr/tid por 11 días) hasta obtener niveles plasmáticos de 0,02 $\mathrm{mg} / \mathrm{L}$ en 2 pruebas separadas por 2 semanas, y no es compatible con la lactancia materna.

Los antiTNF no se han asociado con incremento en el riesgo de malformaciones congénitas o eventos adversos durante el embarazo. Se debe valorar el riesgo - beneficio de su uso, e idealmente descontinuarlos a la semana 30, para evitar el riesgo de infecciones en el neonato. No se recomiendan estos agentes 
durante el periodo de lactancia, por insuficiencia de datos. El uso de tocilizumab y tofacitinib no se recomienda durante el embarazo y lactancia, por insuficiencia de datos suficientes en humanos. El uso de rituximab podría considerarse en caso de actividad severa o manifestaciones extrarticulares de la enfermedad. Se desconoce si el rituximab se excreta en la leche materna, por lo que debe evitarse durante la lactancia.

\section{Bibliografía recomendada:}

Generalidades

1. Raza, et al. Early rheumatoid arthritis is characterized by a distinct and transient synovial fluid cytokine profile of T cell and stromal cell origin. Arthritis Res Ther 2005; 7:R784-95.

2. Van Nies, et al. What is the evidence for the presence of a therapeutic window of opportunity in rheumatoid arthritis? A systematic literature review. Ann Rheum Dis 2013; 229:286-97.

3. Mchold, et al. Very recent onset rheumatoid arthritis: clinical and serological patient characteristics associated with radiographic progression over the first years of disease. Rheumatology (Oxford) 2007;46: 342-9.

4. Gossec, et al. Prognostic factors for remission in early rheumatoid arthritis: a multiparameter prospective study. Ann Rheum Dis 2004; 63:675-8.

Clinimetría y evaluación clínica de la enfermedad

1. Aletha D. New insights into the measurement of disease activity in rheumatoid arthritis. Curr Opin Rheumatol 2015; 27:288-272.
2. Anderssen A. et al. Rheumatoid Arthritis Disease activity Measures: American Collegue of Rheumatology Recomendations for use in Clinical Practice. Arthritis Care and Research 2012; 64: 640-647.

3. Van Riel P. The development of the Disease Activite Score (DAS) and Disease Activity Score using 28 joint count (DAS28). Clin Exp Rheumatol 2014; 32: S65-S77.

4. Iaqnocco A. Responsiveness in Rheumatoid Arthritis. A report from the OMERACT 11: Ultrasound Workshop. J Rheumatol 2014; 41: 379-382.

Glucocorticoides y drogas antiinflamatorias no esteroideas

1. Smolen, et al. The Stop Arthritis Very Early (SAVE) trial, an International multicentre, randomised, double-blind, placebo-controlled trial on glucocorticoids in very early arthritis. Ann Rheum Dis 2010; 69:495-502.

2. Oscanoa-Espinoza T. Seguridad de los antiinflamatorios no esteroideos. Rev Med Inst Mex Seguro Soc 2015; 53:172-9.

3. Bello A, Holt R. Cardiovascular Risk with Non-steroidal Anti-inflammatory Drugs: Clinical Implications. Drug Saf 2014; 37:897-902.

4. Strehl, Buttgereit F. Optimized glucocorticoid therapy: Teaching old drugs new tricks Mol Cell Endocrinol 2013; 380:32-40.

Fármacos modificadores de la artritis reumatoide (FARMEs) convencionales

1. Emery P, Breedveld FC, Lemmel EM, et al; Multinational Leflunomide Study Group. A comparison of the efficacy and

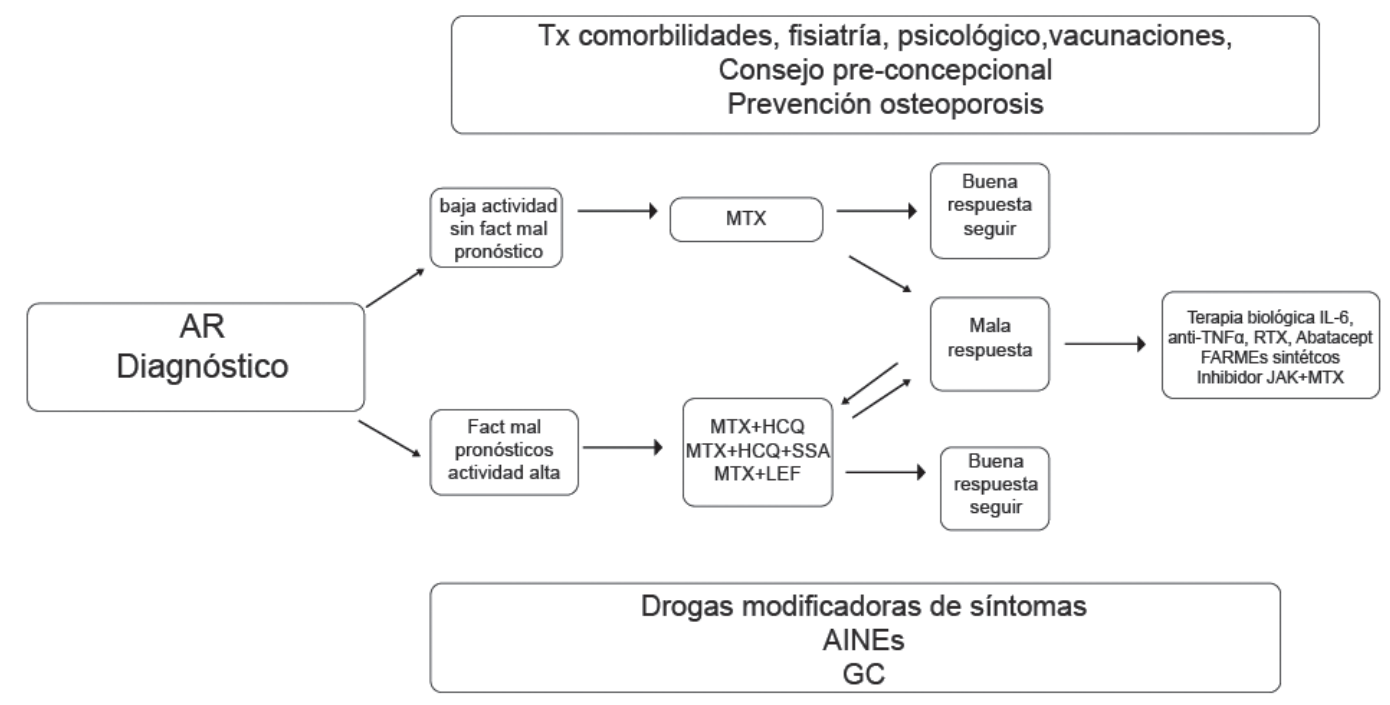

EVOLUCION EN EL TIEMPO

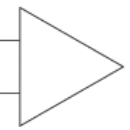

Algoritmo de manejo de la artritis reumatoide 
Guías de manejo de artritis reumatoide / Alpízar-Campos et al

safety of leflunomide and methotrexate for the treatment of rheumatoid arthritis. Rheumatology 2000;39:655-65.

2. Kalden JR, Schattenkirchner M, Sorensen H, et al. The efficacy and safety of leflunomide in patients with active rheumatoid arthritis. Arthritis Rheum 2003;48: 1513-20.

3. Hoekstra M, Haagsma C, Neef C, et al. Bioavailability of higher dose methotrexate comparing oral and subcutaneous administration in patients with rheumatoid arthritis. J Rheumatol 2004; 31:45.

4. Mladenovic V, Domljan Z, Rozinan B, et al. Safety and effectiveness of leflunomide in the treatment of patients with active rheumatoid arthritis. Arthritis Rheum. 1995; 38:1595-1603.

5. Steven J. Katz and. Russell Anthony S. Re-evaluation of antimalarials in treating rheumatic diseases: re-appreciation and insights into new mechanisms of action. Curr Opin Rheumatol 2011; 23:278-281.

Agentes biológicos y moléculas pequeñas

1. Ianculescu I, Weisman N. Infection, malignancy, switching, biosimilars, antibody formation, drug survival and withdrawal, and dose reduction: what have we learned over the last year about tumor necrosis factor inhibitors in rheumatoid arthritis? Curr Opin Rheumatol 2016; 28:303309

2. Jones $\mathrm{G}$, et al. Comparasion of tozilizumab monotherapy versus methotrexate monotherapy in patients with moderate to severe rheumatoid arthritis: the AMBITION Study. Ann Rheum Dis 2010; 69:88-96

3. Cohen $S$, et al. Rituximab for rheumatoid arthritis refractory to anti-tumor necrosis factor therapy. Arthritis Rheum 2006; 54:2793-2806
4. Burmenster G, et al. Tofacitinib (CP-690,550) in combination with methotrexate in patients with active rheumatoid arthritis with an inadequate response to tumour necrosis factor inhibitors: a randomised phase 3 trial. Lancet 2013; 9865:451-460

Consideraciones sobre embarazo y artritis reumatoide

1. Hazes J, et al. Rheumatoid arthritis and pregnancy: evolution of disease activity and pathophysiological considerations for drug use. Rheumatology 2011; 50:1955-1968.

2. Temprano K, Bandlamudi R, Moore T. Antirheumatic Drugs in Pregnancy and Lactation. Semin Arthritis Rheum 2005; 35:112-121.

3. Saavedra M. et al. Guías de práctica clínica para la atención del embarazo en mujeres con enfermedades reumáticas autoinmunes del Colegio Mexicano de Reumatología. Parte II. Reumatol Clin 2015; 11:305-315.

Los siguientes miembros de la Asociación Costarricense de Reumatología participaron en la elaboración de estas Guías de Manejo de la Artritis Reumatoide: Dr. Daniel Alfaro Vargas, Dr. Javier Badilla Umaña, Dr. Francisco Brenes Silesky, Dr. Juan Carlos Contreras Arias, Dra. Ligia Chaverri Oreamuno, Dra. Marisol Cordero Vega, Dra. Evelyn Durán Mora, Dra. Viviana García Torres, Dra. Marianela Guardia Rímolo, Dr. Carlos León Cespedes, Dr. Alexis Méndez Rodríguez, Dr. Erick Molina Guevara, Dr. Adolfo Pacheco Salazar, Dr. Arturo Runnebaum Jiménez, Dr. Claudio Salas Mena, Dr. Marvin Sánchez Benavides. 